\title{
Psychiatrie hospitalière - papier de position de la FMH sur l'évolution du tarif TARPSY
}

Les diverses institutions de psychiatrie hospitalière de tout le pays sont tenues de facturer à l'avenir leurs prestations à l'aide d'un système uniforme de rémunération forfaitaire. Cette exigence demande cependant de relever d'importants défis. Avec TARPSY, il s'agit en effet de développer un système tarifaire le mieux à même de prendre en compte les besoins de la psychiatrie hospitalière. Le groupe d'observation TARPSY de la FMH participe au développement de ce système, et à ce titre, il a défini une prise de position qui énonce les attentes du corps médical.

\section{Bettina Holzer}

Tarifs et économie de la santé pour les médecins hospitaliers

\begin{abstract}
Nouveau modèle tarifaire sous l'égide de la société SwissDRG

Dans le cadre de la révision partielle de la loi fédérale sur l'assurance-maladie (LAMal) dans le domaine du financement hospitalier, le législateur exige un système de rémunération forfaitaire lié aux prestations applicable de manière uniforme à toute la Suisse. Cependant, les exigences posées à une structure tarifaire pour les soins de psychiatrie hospitalière se distinguent de celles du système tarifaire SwissDRG avec lequel les hôpitaux facturent leurs soins somatiques

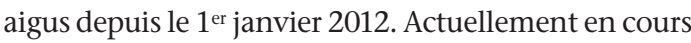
de développement sous l'égide de la société SwissDRG SA, la structure tarifaire nationale pour la psychiatrie hospitalière TARPSY devrait être introduite à l'horizon 2015/2016 [1]. La conduite de ce projet a été confiée à $\mathrm{H}+$ et à la Direction de la santé du canton de Zurich.
\end{abstract}

\section{«Les exigences posées à une structure tarifaire pour les soins de psychiatrie hospitalière se distinguent de celles du système tarifaire SwissDRG.»}

Correspondance: Dr Bettina Holzer FMH

Froburgstrasse 15 CH-4600 Olten Tél. 0313591111 Fax 0313591112 tarife.spital[at]fmh.ch

\section{Eléments cardinaux de la structure tarifaire}

Les travaux préliminaires ont permis de définir les éléments cardinaux de la classification. Le premier consiste à obtenir le moins possible de PCG (Psychiatric Cost Groups), chacun devant être homogène en termes de coûts et défini d'après les groupes de diagnostics principaux selon la CIM-10 GM [2], le second point déterminant de la structure étant la gravité du cas. Le comité de pilotage TARPSY collabore avec l'Association nationale pour le développement de qualité dans les hôpitaux et les cliniques (ANQ) qui, en matière d'assurance-qualité, utilise des instruments d'évaluation par le médecin et d'autoévaluation [3] par le patient pour déterminer la gravité d'un cas, celle-ci permettant de différencier un traitement ordinaire d'un traitement intensif. Par ailleurs, le système tarifaire doit tenir compte des particularités de catégories telles que les enfants et les adolescents ou les patients en milieu carcéral (forensique). Enfin, la structure tarifaire à venir prévoit une combinaison de forfaits par cas et de forfaits journaliers par PCG.

\section{Etat actuel du projet}

Près de 20 cliniques-pilotes spécialisées en psychiatrie de l'adulte, de l'enfant et de l'adolescent participent à la collecte des données 2013. Dès 2014, toutes les institutions de soins psychiatriques seront tenues de fournir leurs données pour le développement ultérieur de TARPSY. Actuellement, la Haute école zurichoise de sciences appliquées (ZHAW) évalue les fichiers de données de 2012. Les résultats devraient être présentés au groupe de travail PCG de TARPSY en octobre 2013. Ce n'est qu'à ce moment-là que la qualité des données fournies permettra de déterminer si la collecte des données et les instruments de mesure nécessitent des adaptations. Sur la base de ces résultats, il faudra également examiner si le système tarifaire répond aux besoins particuliers des enfants et des adolescents, et si d'éventuelles modifications s'imposent quant à la planification du projet. Des logiciels de groupage et des règles d'application de TARPSY devront par ailleurs être développés.

\section{Implication du corps médical}

Elaboré par le groupe d'observation TARPSY de la FMH, le papier de position suivant reflète la position du corps médical et constitue l'argumentaire de base pour défendre le point de vue des médecins dans les différents organes du projet TARPSY, mais également dans le conseil d'administration et les autres commissions de SwissDRG SA. Le Dr Matthias Hilpert, 
médecin adjoint en psychiatrie générale à la Clinique psychiatrique de Königsfelden, et le Dr Jürg UngerKoeppel, médecin-chef du service de psychiatrie de l'enfant et de l'adolescent des Services psychiatriques d'Argovie, représentent la FMH à titre d'observateurs au sein du comité de pilotage du projet TARPSY.

\section{Références}

1 Hilpert M. TARPSY - un nouveau système tarifaire pour la psychiatrie hospitalière. Bull Méd Suisses. 2012;93(49):1811 ou www.fmh.ch $\rightarrow$ Tarifs hospitaliers $\rightarrow$ Publications $\rightarrow 2012 \rightarrow$ TARPSY - Un nouveau système tarifaire pour la psychiatrie hospitalière.
2 ICD $10 \mathrm{GM}$ - Classification statistique internationale des maladies et des problèmes de santé, $10^{e}$ révision, German Modification, base de la codification des diagnostics dans la statistique médicale suisse.

3 www.anq.ch $\rightarrow$ Psychiatrie $\rightarrow$ Psychiatrie pour des adultes $\rightarrow$ Informations de mesure $\rightarrow$ HoNOS (Health of the Nation Outcome Scales, instrument d'évaluation externe) et BSCL (Brief Symptom Check List, instrument d'autoévaluation). www.anq.ch

$\rightarrow$ Psychiatrie $\rightarrow$ Psychiatrie d'enfants et d'adolescents $\rightarrow$ Instruments de mesure $\rightarrow$ HoNOSCA (Health of the Nation Outcome Scales Child and Adolescent, instrument d'évaluation externe) et HoNOS-SR (Health of the Nation Outcome Scales Child and Adolescent-Self Rating, instrument d'autoévaluation).

\section{Papier de position de la FMH sur le système tarifaire Psychiatrie (TARPSY)}

Le présent papier de position énonce le point de vue de la FMH au sujet de l'introduction de la structure tarifaire TARPSY à l'échelle nationale pour la psychiatrie hospitalière. Ce document a été élaboré par le groupe d'observation TARPSY de la FMH dans lequel siègent les représentants médicaux de la psychiatrie de l'adulte ainsi que de la psychiatrie de l'enfant et de l'adolescent. Il a été approuvé par le Comité central et par l'Assemblée des délégués respectivement en mai et en juin 2013.

\section{Garantie et promotion de la qualité}

a. Les médecins mais aussi les processus de traitement doivent répondre à des exigences de qualité; cela implique notamment de définir les procédures thérapeutiques dans le respect des lignes directrices et de les évaluer. Cette qualité doit faire l'objet d'une rémunération adéquate dans le système tarifaire TARPSY.

b. Les patientes et les patients bénéficient de la thérapie requise en fonction de la gravité $d u$ cas pendant la durée de traitement nécessaire.

c. La situation particulière de la psychiatrie de l'enfant et de l'adolescent est prise en compte.

d. La structure tarifaire doit permettre l'octroi et le financement adéquat de congés thérapeutiques.

e. Les cliniques psychiatriques de jour ou de nuit sont intégrées dans le système tarifaire TARPSY.

f. La psychiatrie se délimite clairement de la médecine somatique aiguë, de la médecine psychosomatique et de la réadaptation psychiatrique afin que le patient bénéficie d'un traitement optimal dans une clinique adéquate.

g. Une recherche concomitante est capitale afin de détecter suffisamment tôt les éventuels effets négatifs de la nouvelle structure tarifaire et de mettre en place les mesures nécessaires pour les corriger.

\section{Rémunération en adéquation avec les prestations}

a. Déterminée en fonction des diagnostics et de la gravité du cas (traitement ordinaire ou traitement intensif), la rémunération des traitements psychiatriques dépend de la durée de traitement.

b. L'indemnisation des comorbidités somatiques et des maladies intercurrentes pendant le séjour psychiatrique est garantie.

c. Les procédures thérapeutiques demandant d'importants moyens financiers et personnels font l'objet d'une rémunération adéquate.

d. Le financement des enseignants pour la psychiatrie de l'enfant et de l'adolescent est garanti.

\section{Articles}

\section{interactifs}

Vous souhaitez commenter cet article? II vous suffit pour cela d'utiliser la fonction «Ajouter un commentaire» dans la version en ligne. Vous pouvez également consulter les remarques de vos confrères sous: www.bullmed.ch/ numero-actuel/ articles-interactifs/

e. Les prestations de base fixes nécessaires aux urgences psychiatriques sont financées en adéquation avec les prestations.

f. La rémunération de la formation médicale postgraduée et continue est garantie. Le temps nécessaire à la formation est mis à disposition.

g. La structure tarifaire doit être régulièrement développée afin de tenir compte des progrès actuels de la médecine.

\section{Saisie facilitée des prestations}

a. La charge administrative reste dans des limites raisonnables. La documentation des prestations doit être simple et praticable.

b. La saisie des diagnostics s'effectue sur la base du système de classification CIM de l'Office fédéral de la statistique. Par ailleurs, il faudra examiner le bénéfice que peut procurer l'utilisation supplémentaire du système de classification multiaxiale en psychiatrie de l'enfant et de l'adolescent. 